\title{
Neuropatia óptica isquêmica anterior e poliarterite nodosa: relato de caso
}

\author{
Anterior ischemic optic neuropathy and polyarteritis nodosa: case report
}

\author{
Christie Michelle Graf ${ }^{1}$ \\ Thelma Larocca Skare ${ }^{2}$ \\ Carlos Augusto Moreira ${ }^{3}$
}

Trabalho realizado no serviço de oftalmologia e no serviço de reumatologia do Hospital Universitário Evangélico de Curitiba - HUEC - Curitiba (PR) - Brasil.

${ }^{1}$ Médica residente do Serviço de Oftalmologia do Hospital Universitário Evangélico de Curitiba - HUEC Curitiba (PR) - Brasil.

${ }^{2}$ Chefe da Disciplina de Reumatologia da Faculdade Evangélica do Paraná - FEPAR - Curitiba (PR) - Brasil.

${ }^{3}$ Professor Titular do Departamento de Oftalmologia da FEPAR - Curitiba (PR) - Brasil.

Endereço para correspondência: Christie Michelle Graf. Rua Fernandes de Barros, 1850 - Curitiba (PR) CEP 80040-200

E-mail: chrili@terra.com.br

Recebido para publicação em 30.09.2003

Versão revisada recebida em 27.04.2005

Aprovação em 15.07.2005

\section{RESUMO}

Descreve-se uma paciente com diagnóstico de poliarterite nodosa, em tratamento com citostáticos e corticosteróides que desenvolveu neuropatia óptica isquêmica anterior, uma manifestação considerada bastante rara para esta doença.

Descritores: Poliarterite nodosa/complicações; Poliarterite nodosa/quimioterapia; Corticosteróides/uso terapêutico; Manifestações oculares; Neuropatia óptica isquêmica/ etiologia; Vasculite retiniana/etiologia

\section{INTRODUÇÃO}

A poliarterite nodosa (PAN) é uma vasculite necrotizante que afeta artérias de médio e pequeno calibre albergando o potencial de lesar praticamente qualquer sistema do organismo ${ }^{(1)}$. As manifestações mais comuns desta patologia incluem o envolvimento renal que aparece em $75 \%$ dos casos, mialgias e artralgias que surgem em até $60 \%$, manifestações cutâneas e neurológicas que lesam, respectivamente, $55 \%$ e $50 \%$ dos pacientes ${ }^{(2)}$.

O envolvimento ocular é encontrado em 10 a $20 \%$ dos pacientes com PAN e demonstra uma grande diversidade ${ }^{(3)}$. Fazem parte deste espectro: vasculites retinianas e de vasos da coróide, esclerites necrotizantes, ceratites ulcerativas periféricas, envolvimento conjuntival, lesões isquêmicas do nervo óptico e inflamação de vasos da órbita podendo, em alguns casos, causar exoftalmia ${ }^{(3)}$.

A PAN não tratada é geralmente letal, mas o uso de corticóides e citostáticos melhoram muito o seu prognóstico, principalmente se estes medicamentos forem instituídos de maneira precoce ${ }^{(1)}$. Isto é favorecido por um diagnóstico rápido o qual, por sua vez, fica na dependência do conhecimento das suas nuances de apresentação.

Formas oculares podem ser a manifestação inicial da PAN e, nesta situação, o oftalmologista pode ser o responsável pela inclusão desta vasculite no diagnóstico diferencial facilitando, assim, o seu reconhecimento ${ }^{(4)}$.

Os autores relatam um caso de PAN complicada com neuropatia óptica isquêmica anterior, a qual é considerada uma manifestação bastante rara desta patologia na bibliografia compulsada. Isto dá relevo para a forma de envolvimento oftalmológico encontrada na paciente.

\section{RELATO DE CASO}

RFC, sexo feminino, 26 anos, tem diagnóstico de PAN a 6 meses antes do evento atual quando apresentou quadro de hipertensão arterial, mono- 
neurite multiplex em membro superior $\mathrm{D}$ e inferior $\mathrm{D}$ além de vasculite retiniana com diminuição abrupta da acuidade visual do olho direito. Na época esteve internada no serviço de Reumatologia do HUEC tendo sido submetida à investigação que mostrou: pesquisa de fator antinuclear e sorologias para hepatite $\mathrm{B}, \mathrm{C}$, HIV negativas; complementos (C3 e C4), creatinina e hemograma normais. As provas de atividade inflamatória eram altas com VHS de $84 \mathrm{~mm}$ na primeira hora e proteína C reativa de $96 \mathrm{mg} / \mathrm{l}$ e o parcial de urina mostrava traços de proteína e ++ de cilindros hialinos por campo de grande aumento. Uma angiografia mesentérica e renal mostrou múltiplos microaneurismas na topografia de artérias segmentares intra-renais sendo, portanto, compatível com diagnóstico de PAN (Figura 1).

Foi submetida a tratamento com pulsoterapia de metilprednisolona (1 grama endovenosa por três dias consecutivos) seguida de $80 \mathrm{mg}$ prednisona/dia e instalada em uso de ciclofosfamida, $100 \mathrm{mg} / \mathrm{dia}$, via oral. Houve melhora dos sintomas neurológicos e da atividade renal, porém não se observou reversão da perda visual.

Cinco meses depois, estando em uso de ciclofosfamida na dose supra descrita e de $40 \mathrm{mg}$ de prednisona/dia apresentou diminuição da acuidade visual súbita em olho esquerdo. Ao exame físico a pressão arterial era de 130/80 mmHg. Exame do tórax, abdome e membros eram normais. Uma VHS foi de $17 \mathrm{~mm}$ na $1^{\underline{a}}$ hora e a proteína $C$ reativa de $20,3 \mathrm{mg} / \mathrm{l}$. Creatinina e parcial de urina eram normais. O corticóide foi aumentado para $80 \mathrm{mg}$ de prednisona/dia.

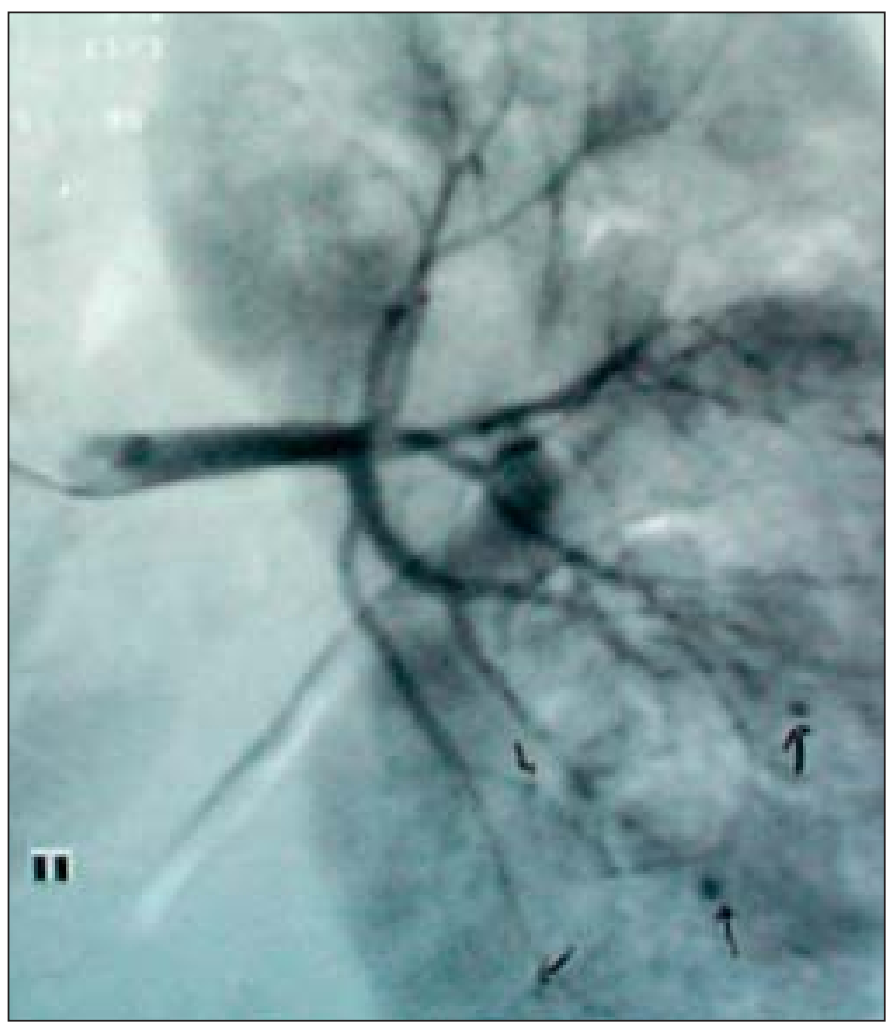

Figura 1 - Arteriografia renal da paciente descrita mostrando microaneurismas típicos da poliarterite nodosa
Ao exame oftalmológico apresentava acuidade visual (AV) de percepção luminosa no olho direito (OD) e 20/400 no olho esquerdo (OE). A biomicroscopia foi normal em ambos os olhos. A oftalmoscopia indireta mostrou atrofia do nervo óptico em olho direito e leve hiperemia de disco óptico à esquerda.

Uma angiofluoresceinografia mostrou perfusão vascular normal, periferia normal e palidez de disco óptico no OD. No OE foi observado hiperemia de disco óptico com hiperfluorescência na fase tardia do exame, o que levou à conclusão diagnóstica de neuropatia óptica isquêmica anterior. Não foi observado zonas "watershed" no exame de angiofluoresceinografia.

A paciente também foi submetida a campimetria computadorizada que revelou perda total de campo visual no OD na escala cinza com "pattern deviation" normal; e resíduo visual paramacular no OE na escala cinza com "pattern deviation" normal. Uma tomografia computadorizada de órbita e de sistema nervoso central foi normal.

Após 10 dias de uso de $80 \mathrm{mg}$ de prednisona/dia a dose deste medicamento foi aumentada para $120 \mathrm{mg} / \mathrm{dia}$ e a de ciclofosfamida para $150 \mathrm{mg} /$ dia.

Revisão após 1 mês de tratamento mostrou que a paciente apresentava acuidade visual de percepção luminosa no OD e 20/200 em OE. Após isto se perdeu o controle por mudança de domicílio.

\section{DISCUSS $\tilde{A} O$}

A neuropatia óptica isquêmica anterior (NOIA) é uma disfunção do nervo óptico, causada pela redução temporária ou permanente na perfusão sanguínea do segmento anterior do nervo óptico ${ }^{(5)}$. Costuma ser didaticamente dividida em aquelas causadas pela arterite temporal ou de células gigantes ou, ainda, NOIA-arteríticas (ou NOIA-A) e as outras NOIA-não arteríticas (ou NOIA-NA) ${ }^{(5)}$.

O segundo grupo NOIA-NA é o mais comum das duas formas e pode ser subdividida em um grupo idiopático (que abrange $70 \%$ dos casos) e um grupo de causas definidas $(30 \%)^{(6)}$. Dentre as causas definidas temos as outras vasculites (como o lupus eritematoso sistêmico, PAN, artrite reumatóide), as vasculopatias sistêmicas (hipertensão arterial, arteriosclerose, diabetes), os distúrbios hemodinâmicos, hematológicos e os oculares (glaucoma simples de ângulo aberto, glaucoma de baixa pressão, pacientes submetidos a facectomia) ${ }^{(5)}$. A NOIANA em pacientes jovens como a da paciente descrita está em geral associada a uma causa definida ${ }^{(7)}$. Entretanto, a NOIA-NA causada por PAN é muito rara. Fujishiro afirma em artigo publicado em 1998 que apenas 4 casos haviam sido descritos no Japão ${ }^{(8)}$.

Nos casos de NOIA-NA, a perda visual é menos severa do que a NOIA-A com uma acuidade visual melhor que 20/200 em $60 \%$ dos casos, o que infelizmente não aconteceu no caso descrito ${ }^{(9)}$.

É interessante notar que pacientes com esta patologia apresentam queixas de acuidade visual pior ao acordar o que é atri- 
buído à hipotensão arterial noturna ${ }^{(9)}$; isto pode ser agravado quando são utilizados medicamentos hipotensores potentes ${ }^{(10)}$.

O edema do disco óptico pode ser difuso ou segmentado, hiperêmico ou pálido. Quando o infarto no nervo óptico já ocorreu há algumas semanas, o edema será gradualmente substituído por atrofia ${ }^{(5)}$. A presença de hemorragias peripapilares em chama de vela e alterações maculares pode aparecer $^{(5)}$. O defeito campimétrico mais comumente encontrado é o altitudinal, mas apenas metade dos casos apresenta defeitos altitudinais inferiores ${ }^{(11)}$.

As características mais comumente observadas na angiografia são a ausência ou diminuição da fluoresceína do disco, o retardo do enchimento do disco óptico, difusa ou setorialmente, sua hiperfluorescência na fase tardia, por extravasamento do corante, e o retardo difuso ou setorial de enchimento da coróide (que ocorre com mais freqüência na forma arterítica) ${ }^{(5)}$.

No caso descrito, chamou a atenção o alto grau de agressividade da doença, uma vez que a NOIA-NA se estabeleceu em OE na vigência do tratamento com ciclofosfamida altas doses de corticóide exigindo aumento destes imunossupressores para doses bastante elevadas.

Os autores concluem que, embora seja uma situação rara, a neuropatia óptica isquêmica anterior deve ser lembrada como pertencente ao espectro das manifestações oculares da poliarterite nodosa.

\section{ABSTRACT}

We describe a patient with polyarteritis nodosa being treated with cytostatics and corticosteroids who developed an ante- rior ischemic optic neuropathy, a rare manifestation of this pathology.

Keywords: Polyarteritis nodosa/complications; Polyarteritis nodosa/drug therapy; Adrenal cortex hormones/therapeutic use; Eye manifestations; Optic neuropathy ischemic/etiology; Retinal vasculitis/etiology

\section{REFERÊNCIAS}

1. Valente RM, O’Duffy JD, Conn DL. Vasculitis and related disorders. In: Kelley WN, Harris ED, Ruddy S, Sledge CB. Textbook of rheumatology. Philadelphia: WB Saunders; 1997. p.1079-132.

2. Skare TL. Vasculitis. In: Skare TL Reumatologia: princípios e práticas. Rio de Janeiro: Guanabara Koogan; 1999. p.158-66.

3. Akova YA, Jabbur NS, Foster CS. Ocular presentation of polyarteritis nodosa. Clinical course and management with steroid and cytotoxic therapy. Ophthalmology. 1998;100(12):1775-81.

4. Hutchinson GH. Polyarteritis nodosa presenting as posterior ischaemic optic neuropathy. J R. Soc Méd. 1984;77(12):1043-6.

5. Oliveira LRM, Dantas AM, Lavinsky J. Neurites ópticas ou neuropatias ópticas inflamatórias. In: Abujamra S. Retina e vítreo: clinica e cirúrgica. São Paulo: Roca; 2000. p.750-66.

6. Lana-Peixoto MA. Anterior ischemic optic neuropathy in children and young adults. Neurology. 1997;48(Suppl):A329

7. Lana-Peixoto MA. Nonarteritic anterior ischemic optic neuropathy of definite etiology. Neurology. 1999;52(Suppl):A32.

8. Fujishiro M, Handa Y, Machino H, Miyata K, Nakajima K, Iwahashi C. [Anterior ischemic optic neuropathy in a case of polyarteritis nodosa]. Ryumachi. 1998;38(6):831-5. Japanese.

9. Arnold CA. Ischemic optic neuropathies. Ophthalmol Clin North Am. 2001;14(1): 83-98.

10. Hayreh SS. Ischaemic optic neuropathy. Indian J Ophthalmol. 2000;48(3):17194. Erratum: Indian J Ophthalmol. 2000;48(4):317.

11. Hattenhauer MG, Leavitt JA, Hodge DO, Grill R, Gray DT. Incidence of nonarteritic anterior ischemic optic neuropathy. Am J Ophthalmol. 1997;123(1): $103-7$.

\section{Curso Certificado de Treinamento Técnico e Científico em Banco de Olhos no Brasil}

\section{3 a 29 de Julho de 2006 São Paulo - SP}

IN FO RMAÇÕ ES: Tel.: (21) 2539-6345

E-mail: apabo@uninet.com.br 